\title{
Pesquisa Participante Baseada na Comunidade: trabalhando com e para latinos rurais no norte da Florida - EUA
}

\author{
Community-Based Participatory Research: working with and for rural Latinos in North \\ Florida - USA
}

\author{
Investigación Participante Basada en la Comunidade: trabajando con y para latinos rurales \\ en el norte del Estado de Florida - EUA
}

\author{
Jeanne-Marie Rodrigues Stacciarini ${ }^{I}$
}

\begin{abstract}
RESUMO: Este relatório cientifíco apresenta uma trajetória de investigação utilizando a abordagem de Pesquisa Participante Baseada na Comunidade quando se trabalha com imigrantes latinos rurais de baixa renda no norte da Flórida, EUA. Esta visão global foca nos estudos desenvolvidos com e para latinos rurais carentes e apresenta uma breve descrição dos objetivos, os principais resultados e as lições aprendidas, a partir dos seis estudos desenvolvidos nos últimos sete anos. Todos os estudos têm sido focados na promoção da saúde mental e sua relação cultural.

Palavras-Chave: Pesquisa baseada na comunidade; imigrantes latinos; saúde mental, relação cultural.
\end{abstract}

\begin{abstract}
This scientific report presents the research trajectory of using Community Based Participatory Research (CBPR) approach when working with low-income, underserved rural Latinos in North Florida, US. This overview focus on the studies developed with and for underserved rural Latinos and presents a brief description of the goals, main findings, and lessons learned from the six studies developed in the past seven years. All the studies have been focused on mental health promotion and its cultural relationship.

Keywords: Community based participatory reaserch; latin immigrating; mental health; cultural relationship.
\end{abstract}

RESUMEN: Este relato científico presenta una línea de investigación utilizando el enfoque de la Encuesta Participante Basada en la Comunidad cuando se trabaja con inmigrantes latinos necesitados y de baja renta que viven en área rural del norte del Estado de Florida, EUA. Esta visión general está centrada en estudios desarrollados con y para latinos de parcos recursos y presenta una breve descripción de los objetivos, los principales resultados, y las lecciones aprendidas con seis estudios realizados durante los últimos siete años. Todos los estudios se han enfocado en la promoción de la salud mental y su relación cultural. Palabras-Clave: Investigación participante basada en la comunidad; inmigrantes latinos; salud mental; relación cultural.

\section{INTRODUÇÃO}

Em relação aos enfermeiros e cientistas, é fundamental lembrar que os resultados finais da pesquisa devem ser as seguintes: melhorar os programas de saúde incluindo os setores público e privado, influenciar as políticas de saúde, melhorar a saúde e o bem-estar das populações diversas, melhorar a qualidade da vida humana, reduzir as disparidades de saúde e divulgar através de revistas científicas e outros fóruns da comunidade.

Pesquisa acoplada à comunidade (PAC) é uma abordagem que valoriza as relações entre as comunidades, os pesquisadores e pesquisa instituições. $\mathrm{Na}$ verdade, membros não são considerados sujeitos da pesquisa; em vez disso, eles são parceiros. PAC é fundada nos princípios de organização comunitária, que deve ser respeitada: conhecer a comunidade, construir a confiança e relacionamentos, fazer parceria com a comunidade, respeitar as culturas e outros fatores da comunidade na concepção de abordagens, ser flexível, liberar o controle para a comunidade e assumir compromissos a longo prazo. De fato, existem diferentes níveis de abordagens de engajamento comunitário, particularmente considerando o envolvimento, o impacto, a confiança e a comunicação com a comunidade, tais como divulgação, consulta, participação, colaboração e liderança compartilhada ${ }^{1}$.

Existem várias abordagens da PAC, e elas têm sido instrumento no processo de compreender as complexidades do ser humano, de perceber as singularidades subjetivas, de valorizar a (co) aprendizagem e preservar a ética na produção do conhecimento científico. Embora o foco desta conferência seja sociopoética, vou discutir uma abordagem de engajamento

${ }^{I}$ Enfermeira. PHD. Faculdade de Énfermagem, Universidade da Flórida, Gainesville, FL, EUA. E-mail: jeannems@ufl.edu IIConferência proferida no ENFCUIDAR, Faculdade de Enfermagem da Universidade do Estado do Rio de Janeiro, 2014. 
de comunidade, intitulada Pesquisa Participante Comunitária (PPC). Embora as diferenças não sejam demasiadamente simplistas, vou apresentar brevemente os principais passos e conceitos sociopoéticos e PPC, nas Figuras 1 e 2, nos seguintes gráficos para diferenciar os passos seguidos por ambas as abordagens: Sociopoética e PPC.

\section{Usando PPC para Saúde Mental}

A PPC tem sido desenvolvida ao longo das últimas décadas como um paradigma de pesquisa transformadora que preenche a lacuna entre a ciência e a prática através de envolvimento com a comunidade e ação social, com o objetivo de aumentar o capital destinado à saúde².

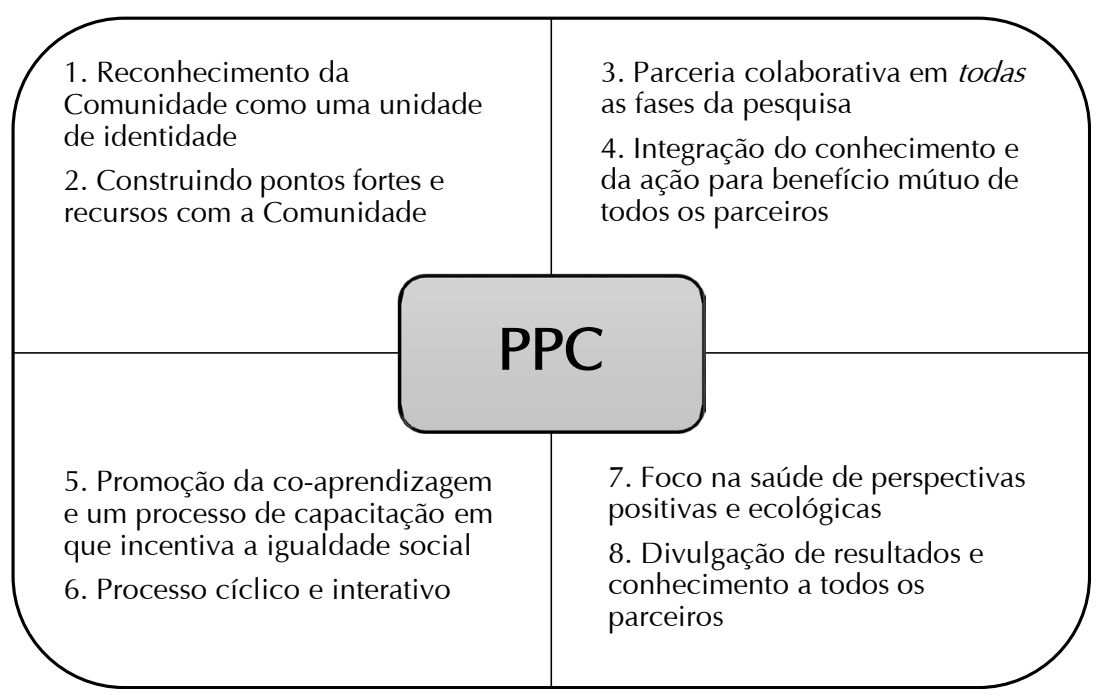

FIGURA 1: Princípios de PPC

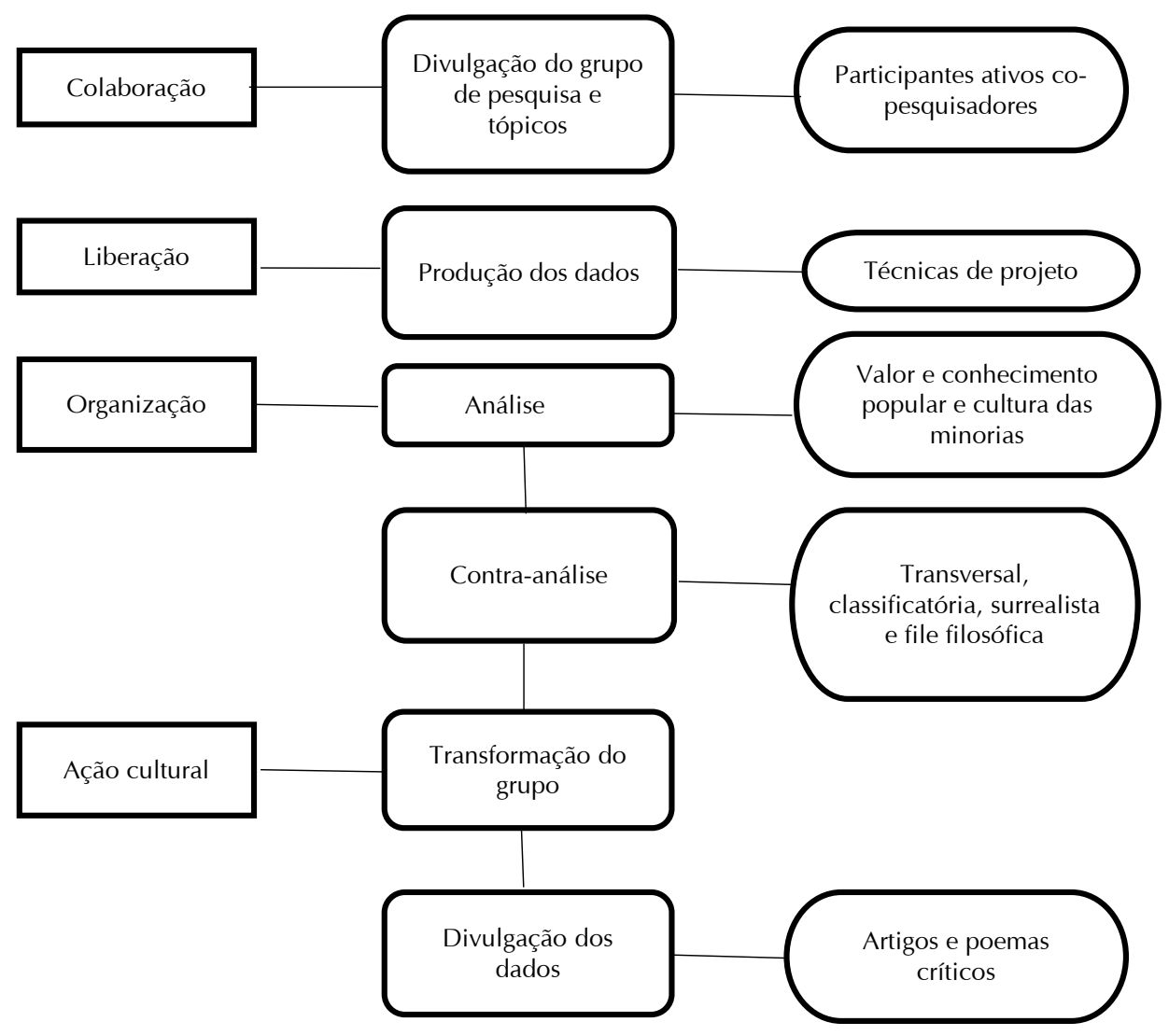

FIGURA 2: Princípios Sociopoéticos 
A PPC foi selecionada como uma abordagem de pesquisa para estudar e interagir com a Comunidade sobre questões de saúde mental em imigrantes latinos que vivem nas zonas rurais da Flórida. Esta abordagem foi escolhida devido ao alinhamento dos princípios de compromisso contínuo dentro da PAC, que nos permitiu desenvolver as intervenções de promoção de saúde mental culturalmente adaptados. A PPC tem suas origens nos estudos de Kurt Lewin ${ }^{3}$, que propôs um programa de pesquisa baseada em ação para as disparidades sociais, e Paulo Freire ${ }^{4,5}$, um educador brasileiro e ativista, que levantou a consciência dos povos oprimidos pelas desigualdades sociais através da educação e engajamento de alfabetização.

A PPC é caracterizada por três Rs: respeito pelos participantes, reconhecimento das contribuições dos participantes e retorno de resultados para a comunidade ${ }^{6}$.

- PPC persegue os seguintes princípios: Colaboração ativa com os participantes da comunidade em todas as fases de pesquisa (a partir do desenvolvimento das questões de pesquisa para divulgação)

- Integração do conhecimento para benefícios mútuos,

- Capacidade de construção e

- Desenvolvimento de recursos dentro da comunidade ${ }^{1}$.

Uma visão geral de todo o trabalho realizado com e para os latinos rurais carentes é apresentada com uma breve descrição do objetivo, principais conclusões e lições aprendidas com os seis estudos que têm sido desenvolvidos nos últimos sete anos. Ver Figura 3.

Todos os estudos foram realizados com os latinos rurais no norte da Flórida, que são considerados novos destinos; ou seja, áreas fora dos portais latinos tradicionais, como Miami, que é um grande centro urbano no sul da Flórida. Essas áreas de novo destino retardam o apoio social dos locais tradicionais, privando muitas vezes imigrantes latinos de recursos sociais significativos ${ }^{7,8}$.

\section{Estudo 1: Convocar um comité de assessoria comunitária (CAC)}

A primeira finalidade do estudo da PPC foi a parceria com a comunidade a fim de realizar o seguinte: desenvolver relacionamentos, informar o processo de investigação, recrutar participantes e recolher dados culturalmente relevantes sobre as necessidades de saúde mental de Latinos em uma comunidade rural. Além disso, o estudo objetivou interpretar dados piloto e projetar estudos futuros com os latinos imigrantes de baixa renda rural no norte da Flórida. Nessa etapa inicial, aprendi uma grande lição do CAC, que eu tinha que seguir as solicitações da comunidade. Nesse caso, apesar das minhas intenções iniciais para incluir apenas as mulheres em meus estudos, eu incluía crianças, a pedido do CAC. Como resultado, novos colaboradores de pesquisa (uma psicóloga infantil e educadora bilíngue) foram convidados a participar do CAC. Dessa forma, fomos capazes de iniciar o processo de construção da parceria para a pesquisa um processo que foi publicado em colaboração com a comunidade e os membros acadêmicos?

\section{Estudo 2: Avaliação de saúde mental}

O objetivo deste estudo foi avaliar sintomas depressivos e estressantes entre uma população rural de baixa renda, mães e filhos latinos e identificar abordagens culturalmente apropriadas ao tratamento que será apoiado pela Comunidade. Embora a abordagem PPC requer o uso de um paradigma ecológico e saudável, nos aproximou dos participantes utilizando a doença

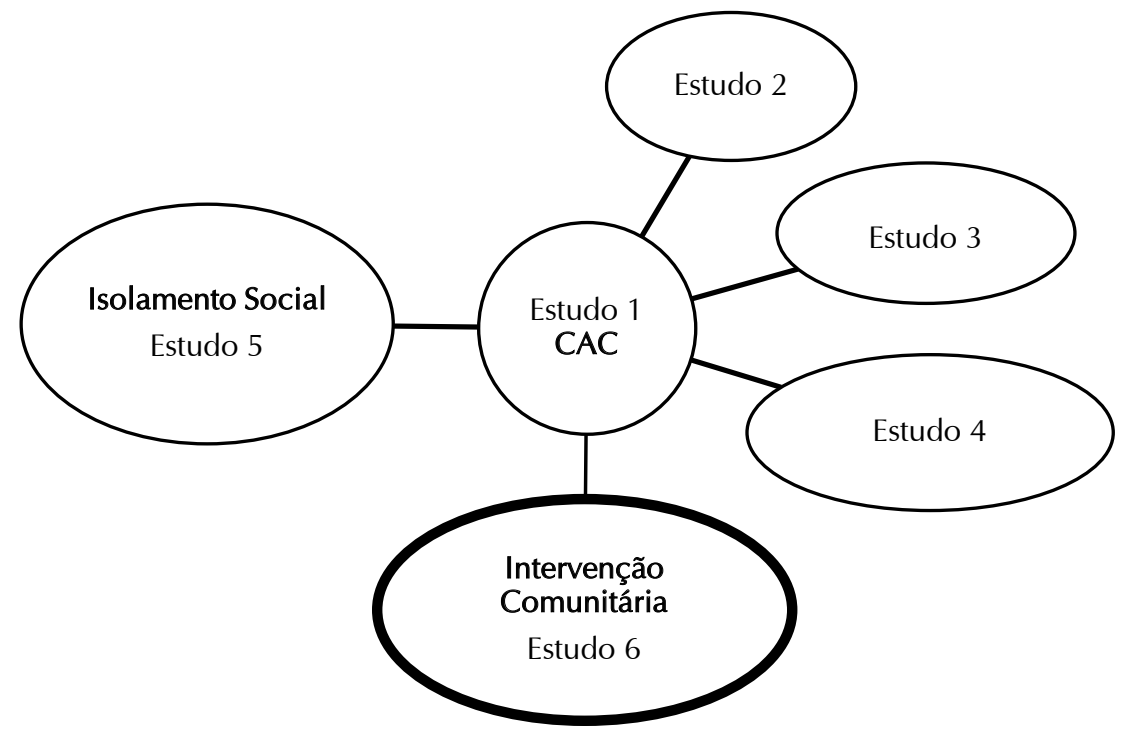

FIGURA 3: Estudos desenvolvidos com imigrantes latinos rurais 
como paradigma e avaliando sintomas depressivos. Nesta etapa, a maior descoberta foi sobre os fenômenos de isolamento social, identificados através de uma questão em aberto no questionário demográfico. Isolamento social pode incluir complexidade psicossocial, linguística e fatores culturais em latinos rurais. Assim, foram desenvolvidos os seguintes estudos para entender mais sobre isolamento social em latinos rurais e identificar as etapas para o desenvolvimento de uma intervenção de base comunitária para minimizar o isolamento social.

\section{Estudo 3: $\mathrm{O}$ envolvimento de organizações baseadas em crenças $(\mathrm{OBC})$}

O objetivo deste estudo foi descrever as percepções dos líderes de $\mathrm{OBC}$ nos contextos psicossociais que afetam o bem-estar mental e possíveis abordagens para a promoção da saúde mental de imigrantes latinos rurais. Os líderes da OBC foram entrevistados e suas percepções foram categorizadas em três níveis diferentes: nível social: saúde e desigualdades sociais e problemas de imigração; nível comunitário: mobilidade geográfica; nível individual: falta de capacidade de construção e de conhecimento linguístico e cultural e personalidades individuais ${ }^{10}$.

Além disso, verificou-se que os líderes de OBC esperam que o envolvimento da comunidade e a unidade cultural surjam como categorias independentes. Os achados desse estudo mostraram que esses líderes são muito bem informados sobre os determinantes psicossociais da saúde mental entre os Latinos rurais. Eles revelaram a necessidade de desenvolver um sistema de laços sociais que se expandem para além de membros da igreja, o que poderia aumentar a coesão da comunidade. Eles também afirmaram que os investigadores precisam entender as redes sociais na comunidade a fim de examinar os fatores que afetam o bem-estar mental. Finalmente, eles notaram que as intervenções comunitárias baseadas na fé//crenças podem ser uma forma segura e culturalmente sensível de intervir junto a imigrantes latinos rurais que estejam em risco para problemas de saúde mental.

\section{Estudo 4: Isolamento social e estudo 5: Am- biente familiar e redes sociais}

O objetivo principal desses estudos foi descrever os membros da família (por exemplo, mães do imigrante latino, pais e adolescentes) as percepções de seu ambiente social rural e isolamento social. Além disso, objetivou-se determinar como esses fatores afetam as suas famílias e bem-estar mental. Através das entrevistas com a família, tornou-se interessante compreender como a ruralidade e as redes sociais atuam como determinantes sociais de saúde dos latinos rurais. Assim, os especialistas acadêmicos adicionais foram procurados para apoiar o desenvolvimento de novos conhecimentos: um geógrafo médico e uma antropóloga com conhecimentos de metodologia de rede social. As conclusões desses estudos mostraram que o isolamento social está relacionado com bem-estar mental dos membros da família ${ }^{11.12}$ bem como de áreas rurais espaciais, particularmente entre as mulheres ${ }^{13}$. Finalmente, isolamento linguístico, racismo, discriminação e medo intenso, devido a sua situação irregular, são fatores que podem afetar negativamente o bem-estar dos latinos rurais.

A Organização Mundial de Saúde considera que todas as profissões devem contribuir para a criação de sociedades mais inclusivas e a promoção do bem-estar através da redução dos determinantes sociais da saúde ${ }^{14}$. Com efeito, nesses estudos, foram aprendidos o valor não tradicional de colaboração interdisciplinar e o engajamento eficaz para a promoção da saúde mental.

\section{Estudo 6: Intervenção comunitária}

Considerando a complexidade dos fatores psicossociais relacionados ao bem-estar mental em latinos rurais, destaca-se o processo de desenvolvimento de uma intervenção nos determinantes sociais da saúde mental (DSSM), que é baseado na igreja e culturalmente adaptado para a comunidade ${ }^{15}$. Para esse estudo, os membros do CAC adaptaram uma intervenção comunitária anteriormente desenvolvida (terapia comunitária) por um psiquiatra social brasileiro - Dr. Barreto. Recentemente, um estudo piloto ( $\mathrm{N}=8$ sessões de grupo) dessa intervenção revelou preocupações adicionais para o psicólogo no seio dessa comunidade: parceiro violento, atitudes passiva, abuso de álcool e drogas e aversão a modalidades ocidentais de tratamento, especialmente para a doença crônica (por exemplo, depressão, diabetes e asma). Análise temática das interações verbais durante as sessões de grupo DSSM demonstra temas principalmente relacionados à falta de acesso de cuidados da saúde, isolamento social e conflito entre os membros da família. Pessoas que aderiram aos consensos, durante as sessões de grupo, foram orientadas a buscar apoio emocional na família ou igreja, bem como a reforçar a perseverança. Elementos culturais implementados (por exemplo, linguagem, configuração de igreja e inclusão de canções latinas tradicionais) aumentam o conforto entre membros e reforçam comportamentos positivos, não verbais. No geral, a intervenção DSSM, que é altamente participativa, permitiu que os membros encontrassem um terreno comum em relação aos complexos determinantes sociais da saúde e desenvolvessem um forte senso de confiança mútua. Tais determinantes serão mais explorados como uma intervenção cultural segura para reduzir o isolamento social de imigrantes latinos rurais.

\section{REFLEXÕES}

A abordagem PPC facilitou uma colaboração ativa e contínua entre acadêmicos e membros da comunidade, permitindo o desenvolvimento de estudos 
que ajudem a promover o bem-estar mental com e para os imigrantes latinos vivendo em áreas rurais do norte da Flórida. Estamos envolvidos em todas as etapas dos estudos de sete pesquisadores e membros de CAC. Além disso, os pesquisadores estiveram envolvidos em vários eventos da comunidade a fim de ser conhecida e confiável para a comunidade-alvo.

Recomendado pelo CAC, toda a coleta de dados foi realizada por um agente comunitário de saúde bilíngue, que é uma promotora. Ela tem sido continuamente treinada em toda a coleta de dados e protocolo de proteção dos direitos humanos pelos pesquisadores. Para ser sensível às necessidades de participantes, toda coleta de dados foi realizada através de reuniões individuais em casa, em dias e horas convenientes.

Vários estudantes de graduação e pós-graduação $(\mathrm{N}=20)$ estiveram envolvidos em projetos diferentes como uma maneira de aprender métodos de pesquisa básica, bem como pesquisas avançadas, tais como, a aplicação de PAC em comunidades carentes. Por causa de seu tempo limitado e também para proteger as comunidades de indivíduos de transição, os alunos têm compromisso limitado com os membros da comunidade. Eles participam de reuniões CAC, eventos da comunidade (ex. feiras de saúde, escola pública eventos) e análise de dados, mas eles não estão envolvidos na coleta de dados e em visitas às casas dos participantes. Para atender aos requisitos de divulgação, as conclusões de todos os estudos têm sido apresentadas em conferências locais, regionais, nacionais e internacionais, bem como publicadas em um diversificado leque de revistas interdisciplinares. Da mesma forma, para atender às necessidades da comunidade, membros de investigação têm participado de vários eventos da comunidade e também apresentaram resultados em diferentes fóruns, tais como igrejas e escolas públicas.

Em nosso ponto de vista, como cientistas, a abordagem PAC provou ser uma oportunidade de pesquisa transformadora para conectar comunidades e acadêmicos, bem como dar às comunidades carentes uma voz genuína em pesquisa e aumentar a probabilidade de sucesso de uma intervenção ${ }^{2,15}$. Além disso, PAC nos tem permitido tornar atores coletivos e criativos, no processo de desenvolvimento, e conceber intervenções culturalmente sensíveis para promover o bem-estar mental com e para as comunidades. Assim, este processo de redução das disparidades de saúde em uma população oprimida tem também transformado os pesquisadores.

\section{REFERÊNCIAS}

1.Department of Health and Human Services (US). Principles of community engagement. Washington (DC): DHHS; 2011.

2.Wallerstein N, Duran B. Community-based participatory research contributions to intervention research: the intersection of science and practice to improve health equity. American Journal of Public Health. 2010; 100 (S1):S40-S6. 3.Lewin K. Resolving social conflicts and field theory in social science. Washington (DC): American Psychological Association; 1997.

4.Freire P. Pedagogy of the oppressed. Penguin: Harmonds Worth; 1972.

5.Freire P. Creating alternative research methods: learning to do it by doing it. New Delhi: Society for Participatory Research in Asia; 1982.

6. Wolf T. The power of collaborative solutions: six principles and effective tools for building healthy communities. San Fransisco. (CA) Jossey Bass; 2010.

7.Shihadeh E, Winters L. Church, place, and crime: Latinos and homicide in new destinations. Sociological Inquiry. 2010; 80:628-49.

8.Shihadeh E, Barranco R. Latino immigration, economic deprivation, and violence: regional differences in the effect of linguistic isolation. Homicide Studies. 2010;14:336-55. 9.Stacciarini JMR, Wiens B, Coady M, Schwait A, Pérez A, Locke B, et al. CBPR: Building partnerships with Latinos in rural area for a wellness approach to mental health. Issues in Mental Health Nursing. 2011;32:486-92.

10. Stacciarini JMR, Vacca R, Wiens B, Loe E, Laflam M, Perez A. Community engaged research: involving FBOs to understand rural Latinos' mental health needs. Cultural Diversity and Ethnic Minority Psychology.2014; in review. 11.Stacciarini JMR, Smith R, Wiens B, Pérez A, Locke B, Laflam M. I didn't ask to come to this country...I was a child: the mental health implications of growing up undocumented. Journal of Immigrant and Minority Health. 2014:1-6.

12.Stacciarini JMR, Smith R, Garvan C, Wiens B, Cottler L. Rural Latinos' mental wellbeing: A mixed-methods pilot study of family, environment and social isolation factors. Community Mental Health Journal. 2014. In review 13.Liang M, Stacciarini JMR, Smith R, Wiens B. Activity space, rurality, and wellbeing of Latino immigrants: a GIS methodology. Health \& Place. 2014; in review.

14. World Health Organization. Closing the gap in a generation: health equity through action on the social determinants of health. Geneva (Swi): Commission on Social Determinants of Health; 2008.

15.Minkler M, Wallerstein N. Community based participatory research for health: process to outcomes. $2^{\text {nd }}$ ed. San Francisco, (CA) Jossey Bass; 2008. 